Document downloaded from:

http://hdl.handle.net/10251/84110

This paper must be cited as:

Esteban Romero, R.; Liriano, O. (2016). Finite groups with all minimal subgroups solitary. Journal of Algebra and Its Applications. 15(8):1650140-1-1650140-9. doi:10.1142/S0219498816501401.

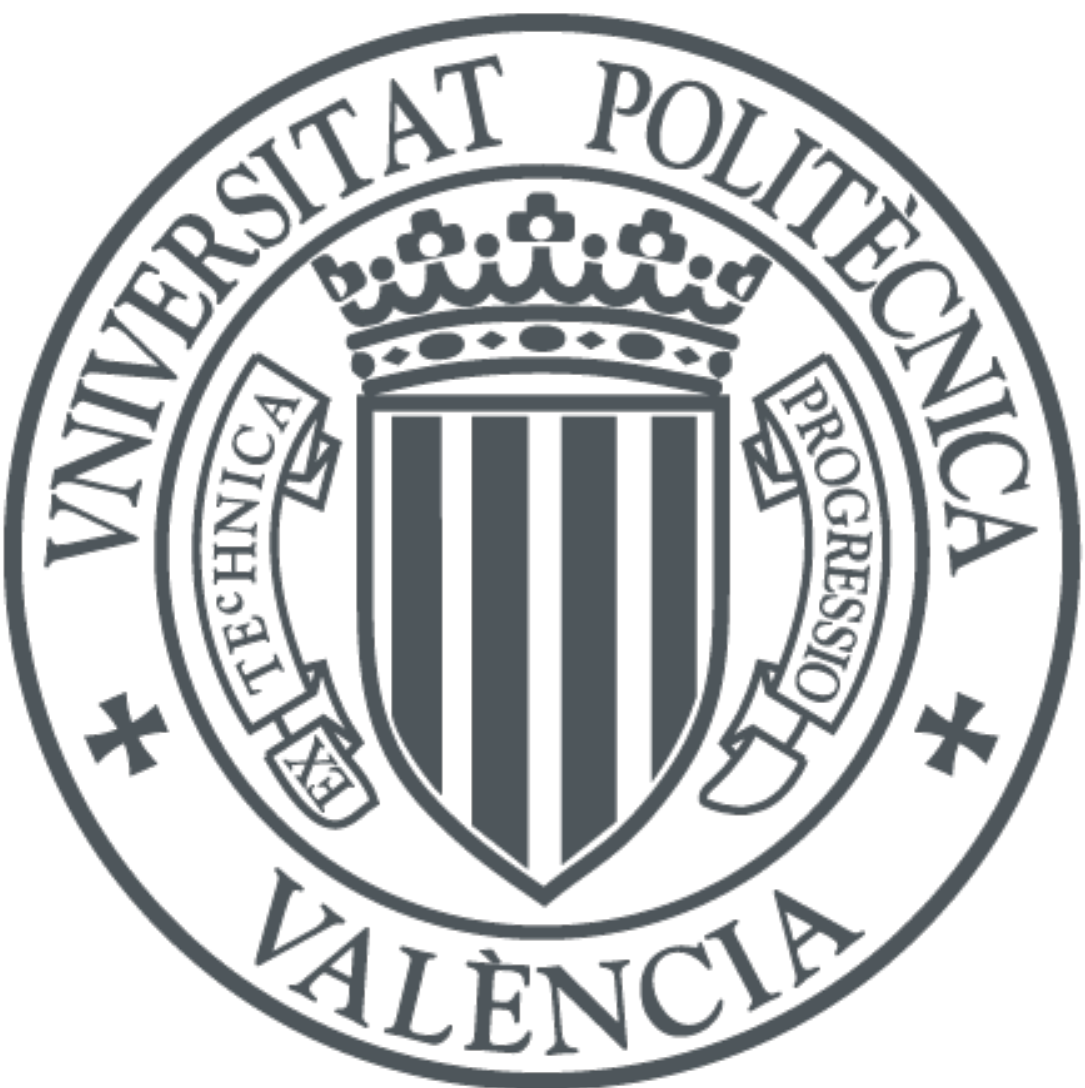

The final publication is available at

http://dx.doi.org/10.1142/S0219498816501401

Copyright World Scientific Publishing

Additional Information 


\title{
Finite groups with all minimal subgroups solitary
}

\author{
R. Esteban-Romero* Orieta Liriano $^{\dagger}$
}

9th October 2015

\begin{abstract}
We give a complete classification of the finite groups with a unique subgroup of order $p$ for each prime $p$ dividing its order.

Mathematics Subject Classification (2010): 20D10, 20D30

Keywords: finite group, solitary subgroup, minimal subgroup
\end{abstract}

\section{Introduction}

All the groups considered in this paper will be finite.

One of the most fruitful lines in the research in abstract group theory during the last years has been the study of groups in which the members of a certain family of subgroups satisfy a certain subgroup embedding property. The family of the subgroups of prime order (also called minimal subgroups) has attracted the interest of many mathematicians. For example, a well known result of Itô ([9], see [8, Kapitel III, Satz 5.3]) states that a group of odd order with all minimal subgroups in the centre is nilpotent. The following result of Gaschütz and Itô ([9], see also [8, Kapitel IV, Satz 5.7]) gives interesting properties of groups with all minimal subgroups normal.

Theorem 1.1. A group $G$ with whose minimal subgroups are normal is soluble and its derived subgroup $G^{\prime}$ possesses a normal Sylow 2-subgroup with nilpotent quotient.

*Institut Universitari de Matemàtica Pura i Aplicada, Universitat Politècnica de València; Camí de Vera, s/n; 46022 València, Spain; email: resteban@mat.upv.es. Current address: Departament d'Àlgebra, Universitat de València; Dr. Moliner, 50; 46100 Burjassot (València), Spain; email: Ramon.Esteban@uv.es.

${ }^{\dagger}$ Departamento de Matemática, Universidad Autónoma de Santo Domingo and Ciclo General, Área de Matemática, Universidad Iberoamericana, Santo Domingo, Dominican Republic; email: ory120@yahoo.com and o.liriano@unibe.edu.do. 
Another result of Buckley [3] states that a group of odd order with all minimal subgroups normal is supersoluble.

A subgroup $H$ of a group $G$ is called solitary when for every subgroup $K$ of $G$ such that $H \cong K$, we have that $H=K$. This concept was introduced by Thévenaz in [12] under the name of strongly characteristic subgroup and studied by Kaplan and Levy in [10]. It is clear that solitary subgroups are characteristic and thus normal.

The aim of this paper is the study of the groups in which all minimal subgroups are solitary. These are the groups $G$ with a unique subgroup of order $p$ for each prime $p$ dividing $|G|$. The starting point is the following well known particular case.

Theorem 1.2 ([8, Kapitel III, Satz 8.2]). Let $p$ be a prime. Suppose that the p-group $G$ has only a subgroup of order $p$.

1. If $p>2$, then $G$ is cyclic.

2. If $p=2$, then $G$ is cyclic or isomorphic to the generalised quaternion group $Q_{2^{s}}$ for an $s \geq 3$, with presentation

$$
Q_{2^{s}}=\left\langle x, y \mid x^{2^{s-1}}=1, x^{2^{s-2}}=y^{2}, x^{y}=x^{-1}\right\rangle .
$$

The following group constructions will play a major role in the classification of groups with all subgroups of prime order solitary. Here $s$ is a natural number with $s \geq 2$.

1. $V(s)=\left[Q_{8}\right] C_{3^{s}}$ and the generator $a$ of $C_{3^{s}}$ acts on $Q_{8}=\langle x, y| x^{4}=$ $\left.1, y^{2}=x^{2}, x^{y}=x^{-1}\right\rangle$ via $x^{a}=y, y^{a}=x y$.

2. $W(s)=\langle a, x, y| a^{3^{s}}=x^{8}=1, y^{2}=x^{4}, x^{y}=x^{-1},\left(x^{2}\right)^{a}=y, y^{a}=$ $\left.x^{2} y, a^{x^{3} y}=a^{-1}\right\rangle$.

Note that the group $W(s)$ has a Sylow 2-subgroup isomorphic to a generalised quaternion group of order 16 and has a normal subgroup of index 2 isomorphic to $V(s)$.

A class of groups is called a formation whenever it is closed under epimorphic images and subdirect products. Given a non-empty formation $\mathfrak{F}$, every group $G$ possesses a smallest normal subgroup $G^{\mathfrak{s}}$, called the $\mathfrak{F}$-residual of $G$, with quotient in $\mathfrak{F}$ (see [2, Section 2.2] for details). It is well known that the classes $\mathfrak{N}$ of all nilpotent groups and $\mathfrak{U}$ of all supersoluble groups are formations. Therefore, every group has a nilpotent residual, which coincides with the last term of the lower central series, and a supersoluble residual.

The first main result of the paper is the following one. 
Theorem 1.3. A group $G$ has all subgroups of prime order solitary if and only if $G$ is a group of the following types:

1. $G=[L] H$, where $H$ and $L$ are cyclic groups of coprime orders, $|L|$ is odd, and all elements of prime order of $H$ centralise $L$.

2. $G=[L](H \times Q)$, where $H$ and $L$ are cyclic groups of coprime odd orders, $Q$ is a generalised quaternion 2-group, all elements of prime order of $H$ centralise $L$, and $Q^{\prime}$ centralises $L$ (in particular, $\left|Q / \mathrm{C}_{Q}(L)\right| \leq 4$ ).

3. $G=[L](H \times V(s))$, where $[Q] C \cong V(s)$, with $L$ and $H$ cyclic $\{2,3\}^{\prime}$ groups of coprime orders, $Q \cong Q_{8}, C \cong C_{3^{s}}, Q$ centralises $L$ and $H$, and all elements of prime order of $H$ and $C$ centralise $L$.

4. $G=[L](H \times W(s))$, where $L$ and and $H$ are cyclic $\{2,3\}^{\prime}$-groups, the elements of prime order of $H$ centralise $L$, and the maximal subgroup $E C$ of $W(s)$ centralises $L$ and $H\left(E=\left\langle x^{2}, y\right\rangle \cong Q_{8}\right.$ and $C=\langle a\rangle \cong$ $C_{3^{s}}$.

Here $[V] X$ denotes the semidirect product of a normal subgroup $V$ acted on by $X$. The notation and the terminology used in this paper agree with [5].

\section{Proof of the main result}

The groups in which all $p$-chief factors for a prime $p$ are cyclic and $G$ isomorphic when regarded as $G$-modules by conjugation have a major role in the proof of the main theorem. We will use the following property of these groups.

Theorem 2.1 (see [1, Theorem 2.1.8]). Let $G$ be a group with nilpotent residual $L$. The following statements are equivalent:

1. For every prime $p$, the $p$-chief factors of $G$ are cyclic and $G$-isomorphic when regarded as modules for $G$ by conjugation.

2. $L$ is an abelian Hall subgroup of odd order of $G$ acted on by conjugation by $G$ as a group of power automorphisms.

Theorem 1.2 shows the interest of the generalised quaternion groups of order $2^{s}$ for a certain $s$ in the scope of our classification. The following lemmas present some well known properties of these groups. They can be found, for example, in [4]. We give proofs of them for the reader's convenience. 
Lemma 2.2. Let $S$ be a non-cyclic subgroup of a generalised quaternion 2-group $Q$. Then $S$ is generalised quaternion.

Proof. Since $S$ is not cyclic, $S$ is not contained in $\langle x\rangle$. Hence there exists an element $x^{u} y \in S \backslash\langle x\rangle$. Moreover, $|S: S \cap\langle x\rangle|=|S\langle x\rangle:\langle x\rangle| \leq 2$. Let $S \cap\langle x\rangle=\left\langle x^{2^{r}}\right\rangle$. It is clear that $S=\left\langle x^{2^{r}}, x^{u} y\right\rangle$ is a generalised quaternion group of order $2^{s-r}$.

Lemma 2.3. The generalised quaternion group $Q=Q_{2^{s}}$ with presentation (1) for $s \geq 4$ does not possess automorphisms of odd prime order.

Proof. If $s \geq 4$, the maximal subgroups $\left\langle x^{2}, y\right\rangle$ and $\left\langle x^{2}, x y\right\rangle$ are not cyclic. By Lemma 2.2, they are isomorphic to the generalised quaternion group $Q_{2^{s-1}}$. Hence the cyclic maximal subgroup $\langle x\rangle$ is a solitary subgroup of $Q$. In particular, $\langle x\rangle \cong C_{2^{s-1}}$ is a characteristic subgroup of $Q$. Let $\alpha$ be an automorphism of $Q$ of odd prime order. Then $\alpha$ can be regarded as an automorphism of $\langle x\rangle$. Since the automorphism group of $C_{2^{s-1}}$ has order $2^{s-2}$ by [8, Kapitel I, Satz 4.6], it follows that $\alpha$ centralises $\langle x\rangle$. On the other hand, $\alpha$ can be regarded as an automorphism of $Q /\langle x\rangle \cong C_{2}$. It follows that $\alpha$ centralises $Q /\langle x\rangle$. By [5, Chapter A, Proposition 12.3], $\alpha$ centralises $Q$. This contradicts the fact that $\alpha$ is an automorphism of $Q$ of odd prime order. The proof of the lemma is then completed.

Lemma 2.4. Let $N$ be a non-cyclic normal subgroup of a generalised quaternion group $Q$. Then $|Q: N| \leq 2$.

Proof. Since $N \not \subset\langle x\rangle$, there exists an element $x^{u} y \in N \backslash\langle x\rangle$. Hence $\left(x^{u} y\right)^{-1}\left(x^{u} y\right)^{x}=y^{-1} x^{-u} x^{-1} x^{u} y x=x^{2} \in N$. It follows that $\left\langle x^{2}\right\rangle$ is a proper subgroup of $N$ and, since $\left|Q:\left\langle x^{2}\right\rangle\right|=4$, the conclusion follows.

Lemma 2.5. If $A / B$ is a non-cyclic elementary abelian section of a generalised quaternion group, then $A / B \cong C_{2} \times C_{2}$.

Proof. Since $A / B$ is a non-cyclic section of $Q, A$ cannot be cyclic. Hence, by Lemma $2.2, A$ is generalised quaternion. Since $A / B$ is elementary abelian, the Frattini subgroup $\Phi(A)$ of $A$ is contained in $B$. But since $A$ is 2-generated, $|A / \Phi(A)|=4$ and so $|A / B| \leq 4$. As $|A / B|>2$, we conclude that $|A / B|=4$ and so $A / B \cong C_{2} \times C_{2}$.

Now we are in a position to prove our main result.

Proof of Theorem 1.3. It is clear that the groups of types 1, 2, 3, and 4 have all subgroups of prime order solitary. 
Assume now that $G$ has all subgroups of prime order solitary. We will prove that $G$ is of one of the types $1,2,3$, or 4 . For the reader's convenience, we break the proof into separately stated steps.

Step 1. G is soluble

Since all solitary subgroups are normal, this claim follows from Theorem 1.1 .

Step 2. Every subgroup of $G$ has all subgroups of prime order solitary.

This is clear.

Now we fix a Hall system $\mathbb{H}$ in $G$, which exists by Step 1 (see [5, Chapter I, Section 4]). If $p$ is a prime and $\pi$ is a set of primes, we will denote by $G_{p}$ the Sylow $p$-subgroup of $G$ in $\mathbb{H}$ and by $G_{\pi}$ the Hall $\pi$-subgroup of $G$ in $\mathbb{H}$.

Step 3. If $p$ is an odd prime, then $G_{p}$ is cyclic. Moreover, $G_{2}$ is cyclic or generalised quaternion.

This is a consequence of Theorem 1.2 and Step 2 .

Step 4. If $|G|$ is not divisible by 3 , then $G$ has no chief factors isomorphic to $C_{2} \times C_{2}$.

Assume that $A / B$ is a chief factor of $G$ isomorphic to $C_{2} \times C_{2}$. Now $G / \mathrm{C}_{G}(A / B)$ is isomorphic to a subgroup of the automorphism group of $C_{2} \times C_{2}$, which is isomorphic to $\mathrm{GL}_{2}(2) \cong \Sigma_{3}$. If 3 does not divide $|G|$, $G / \mathrm{C}_{G}(A / B)$ has order not divisible by 3 and so it is a 2-group. But this is impossible by [5, Chapter A, Proposition 3.12], since $A / B$ is a 2-chief factor of order 4.

Step 5. All chief factors of $G$ are cyclic or isomorphic to $C_{2} \times C_{2}$

Let $p$ be an odd prime. Since $G_{p}$ is cyclic, all $p$-chief factors of $G$ are cyclic. If $G_{2}$ is cyclic, all 2-chief factors of $G$ are cyclic. Suppose that $G_{2}$ is not cyclic. Then $G_{2}$ is generalised quaternion by Theorem 1.2. Given a 2-chief factor $A / B$ of $G$, we have that $A / B$ is isomorphic to an elementary abelian section of $G_{2}$. But every elementary abelian section of $G_{2}$ is a cyclic group or an elementary abelian group of order 4 by Lemma 2.5 .

Step 6. Suppose that $G_{2}$ is cyclic or that $G$ has no chief factors isomorphic to $C_{2} \times C_{2}$. Then $G$ is supersoluble.

This is an immediate consequence of Step 5

Step 7. If $G_{2}$ is cyclic or $G$ has no chief factor isomorphic to $C_{2} \times C_{2}$, then, for every prime $p$, the $p$-chief factors of $G$ are $G$-isomorphic.

By Step 6, $G$ is supersoluble. Suppose that $p_{1}<p_{2}<\cdots<p_{r}$ are the primes dividing $|G|$. Then $G$ has a Sylow tower

$$
1=P_{r} \leq P_{r-1} \leq \cdots \leq P_{1} \leq P_{0}=G
$$

where $P_{i} \unlhd G$ for all $i$ and $P_{i-1} / P_{i}$ is a Sylow $p_{i}$-subgroup of $G / P_{i}$ for $1 \leq i \leq r$. Moreover, $P_{i-1} / P_{i}=G_{p_{i}} P_{i} / P_{i}$ for $1 \leq i \leq r$. We can refine (2) to 
a chief series of $G$. If $G_{p_{i}}=\left\langle a_{i}\right\rangle$, with $\left|G_{p_{i}}\right|=p_{i}^{s_{i}}$, then the chief factors of this series will have the form $\left\langle a_{i}^{p_{i}^{j}}\right\rangle P_{i} /\left\langle a_{i}^{p_{i}^{j+1}}\right\rangle P_{i}$ for $1 \leq i \leq r$ and $0 \leq j \leq s_{i}-1$. Given $g \in G, a_{i}^{g} P_{i}=\left(a_{i} P_{i}\right)^{g P_{i}}=\left(a_{i} P_{i}\right)^{r_{g} P_{i}}=a_{i}^{r_{g}} P_{i}$ for a certain $r_{g} \in \mathbb{Z}$. Hence $\left(a_{i}^{p_{i}^{j}}\right)^{g} P_{i}=\left(a_{i}^{p_{i}^{j}}\right)^{r_{g}} P_{i}$ for $0 \leq j \leq s_{i}-1$. In particular, $\left(a_{i}^{p_{i}^{j}}\right)^{g}\left\langle a_{i}^{p_{i}^{j+1}}\right\rangle P_{i}=$ $\left(a_{i}^{p_{i}^{j}}\right)^{r_{g}}\left\langle a_{i}^{p_{i}^{j+1}}\right\rangle P_{i}$ and $g$ induces the same automorphism of all $p$-chief factors in this series. By the Jordan-Hölder theorem [8, Kapitel I, Satz 11.5], all the $p$-chief factors of $G$ are $G$-isomorphic.

Step 8. $R=G_{\{2,3\}^{\prime}}$ is normal in $G$.

The group $G_{2^{\prime}}$ has all subgroups of prime order solitary and has a trivial Sylow 2-subgroup. By Step $6, G_{2^{\prime}}$ is supersoluble. Since 3 is the smallest prime dividing the order of $G_{2^{\prime}}$, its Hall $3^{\prime}$-subgroup $R$ is normal in $G_{2^{\prime}}$ by [8, Kapitel VI, Satz 9.1]. In particular, $G_{3}$ normalises $R$. On the other hand, the group $G_{3^{\prime}}$ has all subgroups of prime order solitary and has order not divisible by 3 . Hence $G_{3^{\prime}}$ is supersoluble by Step 6 . Therefore its Hall $2^{\prime}$ subgroup $R$ is normal in $G_{3^{\prime}}$ by [8, Kapitel VI, Satz 9.1]. In particular, the Sylow 2-subgroup $G_{2}$ of $G_{3^{\prime}}$ normalises $R$. We conclude that $R$ is normal in $G$.

Step 9. If $G_{2}$ is cyclic or $G$ has no chief factors isomorphic to $C_{2} \times C_{2}$, then the nilpotent residual $L$ of $G$ is a cyclic Hall subgroup of odd order and $G$ induces a group of power automorphisms on $L$.

By the Theorem 2.1, the nilpotent residual $L$ of $G$ is an abelian Hall subgroup of $G$ of odd order. Since the Sylow $p$-subgroups of $L$ are cyclic and the direct product of cyclic subgroups of pairwise coprime orders is cyclic, the result follows.

Let $\pi$ be the set of primes dividing the order of the nilpotent residual $L$ of $G$.

Step 10. If $G_{2}$ is cyclic, then $G$ is a group of type 1 .

Let $H=G_{\pi^{\prime}}$. Since $H \cong G / L, H$ is nilpotent. Now $H$ has all Sylow subgroups cyclic. Hence $H$ is cyclic. Since all subgroups of prime order of $H$ are solitary in $G$, in particular, normal in $G$, and have trivial intersection with $L$, they must centralise $L$. Hence $G=[L] H$ is a group of type 1 .

Step 11. If $G_{2}$ is not cyclic and $G$ has no chief factors isomorphic to $C_{2} \times C_{2}$, then $G$ is a group of type 2 .

Let $D$ be a Hall $\pi^{\prime}$-subgroup of $G$. Note that $D$ is nilpotent, because $D \cong G / L$. Let $H$ be the Hall $2^{\prime}$-subgroup of $D$. Since $H$ is the direct product of cyclic groups of coprime orders, $H$ itself is cyclic. Then $D$ is the direct product of $H$ and a generalised quaternion 2-group $Q$. Moreover, since all subgroups of prime order of $H$ are solitary in $G$, in particular, normal in $G$, and have trivial intersection with $L$, they centralise $L$. On the other 
hand, $Q / \mathrm{C}_{Q}(L)$ can be regarded as a group of automorphisms of $L$. Since this automorphism group is abelian, we obtain that $Q^{\prime} \leq \mathrm{C}_{Q}(L)$.

Step 12. Assume that $G$ is not supersoluble. Then $T=G_{\{2,3\}}$ has a unique chief factor of order $4, \mathrm{O}_{2^{\prime}, 2}(T) / \mathrm{O}_{2^{\prime}}(T) \cong Q_{8}$, and $T / \mathrm{O}_{2^{\prime}, 2}(T)$ is isomorphic to $C_{3}$ or $\Sigma_{3}$.

Let $T=G_{\{2,3\}}$. Note that $T$ and $G / R$ are $T$-isomorphic and so the chief factors of $T$ can be identified with the chief factors of $G$ above $R$.

Consider a chief series

$$
1=T_{0}<T_{1}<T_{2}<\cdots<T_{m-1}<T_{m}=T
$$

of $T$. By intersecting the members of this series with $Q=G_{2}$, we obtain a normal series

$$
1=T_{0} \cap Q \leq T_{1} \cap Q \leq T_{2} \cap Q \leq \cdots \leq T_{m-1} \cap Q \leq T_{m} \cap Q=Q
$$

of $Q$. Suppose that $T_{d} / T_{d-1}$ is a chief factor of order 4 , then the section $\left(T_{d} \cap Q\right) /\left(T_{d-1} \cap Q\right)$ is isomorphic to $C_{2} \times C_{2}$, and that among all these chief factors of order 4 , we choose $d$ as the largest possible index. Hence $T_{d} \cap Q$ is a non-cyclic normal subgroup of $Q$. By Lemma 2.2, $T_{d} \cap Q$ is a generalised quaternion group. Since $\left|\left(T_{d} \cap Q\right) /\left(T_{d-1} \cap Q\right)\right|=4$, we have that $T_{d-1} \cap Q$ is cyclic by Lemma 2.4. It follows that the series (4) contains a unique factor $\left(T_{d} \cap Q\right) /\left(T_{d-1} \cap Q\right)$ of order 4 . Consequently, the series (3) contains a unique chief factor $T_{d} / T_{d-1}$ of order 4 . Moreover, by Lemma 2.4, we have that $\left|Q: T_{d} \cap Q\right| \leq 2$. Let $U=\mathrm{C}_{T}\left(T_{d} / T_{d-1}\right)$. Then $U$ is a normal subgroup of $T$ and $T / U$ is isomorphic to a subgroup of the symmetric group $\Sigma_{3}$ of degree 3 containing the corresponding alternating group $A_{3}$. Moreover, all other 2-chief factors of $T$ are central. Since $\mathrm{O}_{2^{\prime}, 2}(T)$ is the intersection of the centralisers of all 2-chief factors of $G$ by [5, Chapter A, Theorem 13.8], we obtain that $\mathrm{O}_{2^{\prime}, 2}(T)=U$. Let $Y=\mathrm{O}_{2^{\prime}}(T)$. Since $T / U$ has a subgroup of order 3 , the group $U / Y \cong T_{d} \cap Q$ has an automorphism of order 3 (note that this subgroup of order 3 cannot centralise the chief factor $T_{d} / T_{d-1}$ ). By Lemma $2.3, U / Y \cong Q_{8}$.

Step 13. Assume that $G$ is not supersoluble. Let $T=G_{\{2,3\}}$ and $U=$ $\mathrm{O}_{2^{\prime}, 2}(T)$. If $T / U \cong C_{3}$, then $T \cong V(s)$ for a certain $s \geq 2$.

Suppose first that $T / U \cong C_{3}$. Let $C=G_{3}=\langle a\rangle \cong C_{3^{s}}$. The element $a^{3}$ belongs to $U$ and hence it belongs to its Sylow 3-subgroup $Y=\mathrm{O}_{3}(T)$. Therefore $Y=\Phi(C)$. Since $Y$ is a normal subgroup of $T$, by [5, Chapter A, Theorem 9.2(d)], $Y \leq \Phi(G)$. But $T / Y$ is a 3-nilpotent group. It follows that $T$ is a 3-nilpotent group, since the class of all 3-nilpotent groups is closed under Frattini extensions (see [8, Kapitel VI, Hilfssatz 6.3]). Therefore the 
Sylow 2-subgroup $Q=G_{2}$ of $G$ is normal in $T$ and is complemented by the cyclic Sylow 3 -subgroup $C \cong C_{3^{s}}$. Hence $T \cong V(s)$.

Step 14. Assume that $G$ is not supersoluble. Let $T=G_{\{2,3\}}$ and $U=$ $\mathrm{O}_{2^{\prime}, 2}(T)$. If $T / U \cong \Sigma_{3}$, then $T \cong W(s)$ for a certain $s \geq 2$.

Suppose now that $T / U \cong \Sigma_{3}$. Let $M / U$ be the Sylow 3-subgroup of $T / U$. In this case, the Sylow 2-subgroup $Q=G_{2}$ of $G$ is isomorphic to a generalised quaternion group of order 16 . We can suppose that $Q=\langle x, y|$ $\left.x^{8}=1, y^{2}=x^{4}, x^{y}=x^{-1}\right\rangle$ and that $a$ is a generator of the Sylow 3-subgroup $C=G_{3}$; moreover, the subgroup $\left\langle x^{4}\right\rangle$ of order 2 is a normal subgroup of $T$ contained in $\Phi(Q)$ and so $\left\langle x^{4}\right\rangle \leq \Phi(T)$ by [5, Chapter A, Theorem 9.2(d)], and the element $a^{3}$ belongs to $U$, hence it belongs to its unique Sylow 3subgroup $Y=\mathrm{O}_{3}(M)$, and so $Y \leq \Phi(T)$. It follows that the supersoluble residual of $T / \Phi(T)$ is an elementary abelian normal subgroup of order 4 and so it is complemented by a subgroup $D / \Phi(T) \cong \Sigma_{3}$ by [2, Theorem 4.2.17], and that all such complements are conjugate. By the conjugacy, we can assume that a Sylow 2-subgroup of $D / \Phi(T)$ is contained in the Sylow 2subgroup $Q \Phi(T) / \Phi(T)$ and that $a \in D$. Hence there exists an element $z \in(Q \cap D) \backslash U$ and $a^{z} \Phi(T)=a^{-1} \Phi(T)$, that is, $a^{z} a \in \Phi(T)$. We may assume that $E=\left\langle x^{2}, y\right\rangle$ is the Sylow 2-subgroup of $M$ and $a$ acts on $E$ via $\left(x^{2}\right)^{a}=y$, $y^{a}=x^{2} y$. Since $z^{2} \in \Phi(T)$ and $z$ is not the element of order 2 of $T$ because $z \neq x^{4}, z$ is an element of order 4 . Furthermore, $z \notin E$. Consequently, we have that $z=x^{1+2 e} y$ for a certain integer $e$. On the other hand, since $a^{z} a \in \Phi(T)$, this element centralises $E$. Note that $y^{x}=x^{-1} y x=x^{-2} y$. Hence $y=y^{z^{-1} a z a}=y^{y^{-1} x^{-1-2 e} a x^{1+2 e} y a}=\left(x^{2(1+2 e)} y\right)^{a x^{1+2 e} y a}=\left(y^{(1+2 e)} x^{2} y\right)^{x^{1+2 e} y a}=$ $\left(y^{2+2 e} y^{-1} x^{2} y\right)^{x^{1+2 e} y a}=\left(x^{4+4 e} x^{-2}\right)^{x^{1+e} y a}=\left(x^{2+4 e}\right)^{y a}=\left(x^{-2-4 e}\right)^{a}=y^{-1-2 e}$, which implies that $e \equiv 3(\bmod 4)$. This gives the presentation of $W(s)$.

Step 15. Suppose that $G_{\{2,3\}} \cong V(s)$. Then $G$ is a group of type 3 .

Let $Q=G_{2}$ and $C=G_{3}$, then $Q \cong Q_{8}, C \cong C_{3}$, and $Q C \cong V(s)$. Clearly, the Hall $2^{\prime}$-subgroup $G_{2^{\prime}}=R C$ is a group of type 1 and so $R C=$ $[L](H \times C)$, where $H$ and $L$ are cyclic $\{2,3\}^{\prime}$-groups of coprime orders, and all elements of prime order of $H C$ centralise $L$. In particular, the elements of prime order of $H$ and the elements of prime order of $C$ centralise $L$. Now $Q C$ acts on all $G$-chief factors of $R$, which are cyclic. Therefore its derived subgroup $(Q C)^{\prime}=Q$ centralises all $G$-chief factors of $R$. By [5, Chapter A, Proposition 12.3], $Q$ centralises $R$.

Step 16. Suppose that $G_{2,3} \cong W(s)$. Then $G$ is a group of type 4 .

The Hall $3^{\prime}$-subgroup $G_{3^{\prime}}=R Q$ of $G$ is a group of type 2. Therefore $G=$ $[L](H \times Q)$, where $H$ and $L$ are cyclic $\{2,3\}^{\prime}$-groups of coprime orders, and all elements of prime order of $H$ centralise $L$. Moreover, all the $G$-chief factors of $R$ are cyclic. Hence the derived subgroup $E C$ of $G_{\{2,3\}}$ centralises all the 
$G$-chief factors of $R$. By [5, Chapter A, Proposition 12.4], $E C$ centralises $L$ and $H$. It follows that $G$ is a group of type 4 .

\section{Final remarks}

Schmidt [11] has studied the groups with a unique subgroup of order $p$ for a given odd prime $p$.

Theorem 3.1 (Schmidt [11, Theorem 7]). Let $p$ be an odd prime. Every finite group with a unique subgroup of order $p$ is an extension of a normal p-nilpotent subgroup with a unique subgroup of order $p$ by a cyclic quotient of order dividing $p-1$ and acting faithfully, and, conversely, every such extension has a unique subgroup of order $p$. In particular, every such group is p-soluble of p-length equal to 1 .

The proof of Theorem 3.1 depends on the well known p-nilpotency criterion of Burnside and a generalisation of this criterion (see, for instance, [7, Theorem 7.4.3 and Theorem 7.4.4]). This result can be used to prove an alternative proof of the solubility of the groups with all minimal subgroups solitary (Step 1 of the proof of Theorem 1.3).

We might also consider the dual problem of classifying the groups with all maximal subgroups solitary. It is clear that a group with all maximal subgroups solitary must be nilpotent, because in this case all maximal subgroups are normal (see [5, Chapter A, Theorem 8.3]). The following examples obtained with the help of the computer algebra system GAP [6] seem to indicate that the classification of the $p$-groups with all maximal subgroups solitary, for a prime $p$, is a difficult problem.

Example 3.2. The quasidihedral group

$$
\mathrm{QD}_{16}=\left\langle a, b \mid a^{8}=b^{2}=1, a^{b}=a^{3}\right\rangle
$$

has three maximal subgroups $\left\langle a b^{-1}, a^{2}\right\rangle \cong Q_{8},\left\langle b, a^{2}\right\rangle \cong D_{8},\langle a\rangle \cong C_{8}$. Hence all of them are solitary.

Example 3.3. The group $G=C_{8} \times \mathrm{QD}_{16}$ has seven maximal subgroups, all of them solitary in $G$.

Example 3.4. The group $G=\langle a, b, c, d, e, f| c=[b, a], d=[c, a], e=$ $[c, b], f=[d, a], a^{3}=c^{-3}=f, b^{3}=d^{3}=e^{3}=f^{3}=1,[d, a]=[e, a]=[d, b]=$ $[e, b]=f,[f, b]=[f, d]=[f, e]=[d, c]=[e, c]=[e, d]=1\rangle$, corresponding to SmallGroup $(729,41)$ in the library of groups of small order of GAP [6], is another example of a 2-generated non-cyclic 3-group in which its four maximal subgroups are solitary. 


\section{Acknowledgements}

The first author has been supported by the research grant MTM2014-54707C03-1-P from the Ministerio de Economía y Competitividad, Spain. The research of the second author has been done during some visits to the Departament de Matemàtica Aplicada and the Institut de Matemàtica Pura i Aplicada of the Universitat Politècnica de València and the Departament d'Àlgebra of the Universitat de València. She expresses her gratitude to these institutions for the use of their facilities and, especially to the first university for the financial support of some of the visits.

The authors thank the anonymous referees for their helpful comments, that have been useful to improve the exposition of this paper, as well as Adolfo Ballester-Bolinches and Jack Schmidt for some conversations concerning this subject.

\section{References}

[1] A. Ballester-Bolinches, R. Esteban-Romero, and M. Asaad. Products of finite groups, volume 53 of de Gruyter Expositions in Mathematics. Walter de Gruyter, Berlin, 2010.

[2] A. Ballester-Bolinches and L. M. Ezquerro. Classes of Finite Groups, volume 584 of Mathematics and its Applications. Springer, New York, 2006 .

[3] J. Buckley. Finite groups whose minimal subgroups are normal. Math. Z., 116:15-17, 1970.

[4] K. Conrad. Generalized quaternions. http://www. math.uconn.edu/ rkconrad/blurbs/grouptheory/genquat.pdf, visited 22 August 2015, 2013.

[5] K. Doerk and T. Hawkes. Finite Soluble Groups, volume 4 of De Gruyter Expositions in Mathematics. Walter de Gruyter, Berlin, New York, 1992.

[6] The GAP Group. GAP - Groups, Algorithms, and Programming, Version 4.7.8, 2015. http: //www.gap-system.org.

[7] D. Gorenstein. Finite Groups. Chelsea Pub. Co., New York, 1980.

[8] B. Huppert. Endliche Gruppen I, volume 134 of Grund. Math. Wiss. Springer Verlag, Berlin, Heidelberg, New York, 1967. 
[9] N. Itô. Über eine zur Frattini-Gruppe duale Bildung. Nagoya Math. J., 62:400-401, 1955.

[10] G. Kaplan and D. Levy. Solitary subgroups. Comm. Algebra, 37(6):1873-1883, 2009.

[11] J. Schmidt. Finite groups with a unique subgroup of order $p$. URL http: //www.ms . uky . edu/ jack/2009-07-08-UniqueSubgroupOfOrderP. pdf, visited 22 August 2015, 2009.

[12] J. Thévenaz. Most finite groups are p-nilpotent. Expo. Math., 11(4):359363, 1993. 